\title{
Avaliação da sensibilidade dos métodos direto à fresco e Hoffman para Ascaris
}

\section{Lumbricoides}

\author{
Sensitivity evaluation of direct fresh and Hoffman methods for Ascaris Lumbricoides
}

Evaluación de sensibilidad de métodos frescos directos y de Hoffman para Ascaris Lumbricoides

Isaias Pinheiro da Cunha

ORCID: https://orcid.org/0000-0003-3613-197X Universidade Nilton Lins, Brasil

E-mail: isaiaspinheiro09@gmail.com

Omero Martins Rodrigues Junior

ORCID: https://orcid.org/0000-0002-8552-3278

Universidade Nilton Lins, Brasil

E-mail: omeromartins.farma@gmail.com

\begin{abstract}
Resumo
O diagnóstico laboratorial da ascaridíase se dá através do Exame Parasitológico de Fezes (EPF), onde é pesquisado a presença de ovos do Ascaris lumbricoides nas fezes. O Método Hoffman se fundamenta na sedimentação espontânea e permite a concentração de ovos, cistos, oocistos e larvas de inúmeras espécies por meio de uma sedimentação gravitacional de uma amostra fecal. E o Exame Direto a Fresco, permite a identificação de protozoários (trofozoítas e cistos) e de helmintos (ovos, larvas e pequenos adultos). Objetivo: Comparar a sensibilidade dos métodos laboratoriais direto a fresco e de Hoffman na área de Parasitologia. Metodologia: A busca de artigos científicos foi realizada nas bases de dados eletrônicas: Scientific Electronic Library Online (SCIELO), Literatura Latino-Americana em Ciências da Saúde (LILACS), National Library of Medicine and the National Institute of Health (Pubmed). Publicações de 2015 a 2021, em idiomas português, espanhol e inglês. Resultados e Discussão: O exame direto a fresco e a técnica de Hoffman, Pons e Janer ou Lutz (HPJL) são os métodos laboratoriais mais utilizados, pois estas técnicas têm como principal vantagem a necessidade mínima de materiais e recursos financeiros. Porém a desvantagem é de apresentar uma grande quantidade de detritos fecais no sedimento, dificultando a preparação e o exame microscópico. Considerações Finais: Direto a fresco e Hofmman, quando utilizados em conjunto aumentam as probabilidades de diagnósticos reais e confiáveis, principalmente no diagnóstico de ovos e cistos de helmintos, porém separados cada um possui maior sensibilidade em casos específicos. Pela escassez de estudos comparativos destes dois métodos, fica evidente e vale a sugestão de mais pesquisas nesse sentido, uma vez que estes métodos se apresentam tão importantes no diagnóstico das parasitoses, que têm se tornado uma questão de saúde pública, porém pelo difícil diagnóstico acaba sendo negligenciada no nosso dia a dia.
\end{abstract}

Palavras-chave: Direto a fresco; Hoffman; Sedimentação espontânea; Sensibilidade.

\begin{abstract}
The laboratory diagnosis of ascariasis is done through the Parasitological Examination of Feces (EPF), which investigates the presence of Ascaris lumbricoides eggs in the feces. The Hoffman Method is based on spontaneous sedimentation and allows the concentration of eggs, cysts, oocysts and larvae of numerous species through gravitational sedimentation of a fecal sample. And the Direct Fresh Examination allows the identification of protozoa (trophozoites and cysts) and helminths (eggs, larvae and small adults). Objective: To compare the sensitivity of directfresh and Hoffman laboratory methods in the field of Parasitology. Methodology: The search for scientific articles was performed in electronic databases: Scientific Electronic Library Online (SCIELO), Latin American Literature on Health Sciences (LILACS), National Library of Medicine and the National Institute of Health (Pubmed). Publications from 2015 to 2021, in Portuguese, Spanish and English. Results and Discussion: Direct fresh examination and the technique of Hoffman, Pons and Janer or Lutz (HPJL) are the most used laboratory methods, as these techniques have as main advantage the minimal need for materials and financial resources. However, the disadvantage is that there is a large amount of fecal debris in the sediment, making preparation and microscopic examination difficult. Final Considerations: Fresh Direct and Hofmman, when used together, increase the probability of real and reliable diagnoses, especially in the diagnosis of helminth eggs and cysts, but separated, each has greater sensitivity in specific cases. Due to the scarcity of comparative studies of these two methods, it is evident and worth suggesting further research in this regard, since these methods are so important in the diagnosis of parasitic diseases, which have become a public health issue, but due to the difficult diagnosis it ends up being neglected in our day to day.
\end{abstract}

Keywords: Straight to fresh; Hoffman; Spontaneous sedimentation; Sensitivity. 


\begin{abstract}
Resumen
El diagnóstico de laboratorio de ascariasis se realiza a través del Examen Parasitológico de Heces (EPF), que investiga la presencia de huevos de Ascaris lumbricoides en las heces. El Método Hoffman se basa en la sedimentación espontánea y permite la concentración de huevos, quistes, ooquistes y larvas de numerosas especies mediante la sedimentación gravitacional de una muestra fecal. Y el examen directo en fresco permite la identificación de protozoos (trofozoítos y quistes) y helmintos (huevos, larvas y pequeños adultos). Objetivo: Comparar la sensibilidad de los métodos de laboratorio de fresco directo y Hoffman en el campo de la parasitología. Metodología: La búsqueda de artículos científicos se realizó en bases de datos electrónicas: Scientific Electronic Library Online (SCIELO), Literatura Latinoamericana en Ciencias de la Salud (LILACS), Biblioteca Nacional de Medicina e Instituto Nacional de Salud (Pubmed). Publicaciones de 2015 a 2021, en portugués, español e inglés. Resultados y Discusión: El examen fresco directo y la técnica de Hoffman, Pons y Janer o Lutz (HPJL) son los métodos de laboratorio más utilizados, ya que estas técnicas tienen como principal ventaja la mínima necesidad de materiales y recursos económicos. Sin embargo, la desventaja es que hay una gran cantidad de desechos fecales en el sedimento, lo que dificulta la preparación y el examen microscópico. Consideraciones finales: Fresh Direct y Hofmman, cuando se usan juntos, aumentan la probabilidad de diagnósticos reales y confiables, especialmente en el diagnóstico de huevos y quistes de helmintos, pero separados, cada uno tiene mayor sensibilidad en casos específicos. Debido a la escasez de estudios comparativos de estos dos métodos, es evidente y vale la pena sugerir una mayor investigación al respecto, ya que estos métodos son tan importantes en el diagnóstico de enfermedades parasitarias, que se han convertido en un problema de salud pública, pero debido a la dificultad. diagnóstico termina siendo descuidado en nuestro día a día.
\end{abstract}

Palabras clave: Directo a fresco; Hoffman; Sedimentación espontánea; Sensibilidad.

\title{
1. Introdução
}

Os parasitos intestinais se configuram como um grave problema de saúde pública, principalmente em países subdesenvolvidos, onde o sistema de saneamento básico não é acessível a toda a população (Munareto et al., 2021). As crianças estão no grupo mais vulnerável à infestação por doenças parasitárias e oportunistas, principalmente a Ascaridíase, pois elas não possuem hábitos adequados de higiene pessoal se expondo ao solo e à água, principais fatores de contaminação (Pinto et al., 2018).

A Ascaridíase é uma doença parasitária humana, causada por um helminto, o Ascaris lumbricoides, geralmente sem sintomatologia, mas quando em grande número pode manifestar-se por dor abdominal, diarréia, náuseas, anorexia, causando obstrução intestinal e muitas vezes infestação pulmonar (Texeira et al., 2019).

A infecção pelo Ascaris pode ser recorrente, pois se for infectado uma vez não garante imunidade do indivíduo perante o parasita, sendo viável uma possível reinfecção ao mesmo individuo desenvolvendo a parasitose ao longo da vida. O Ascaris Lumbricoide quando adulto pode atingir de 20 a $30 \mathrm{~cm}$ de comprimento e demonstram uma cor leitosa. O vestíbulo bucal está localizado na extremidade anterior, e é contornado por três fortes lábios assemelha-se de dentículos e sem interlábios (Antunes \& Libardoni, 2017). Segundo a Organização Mundial da Saúde (OMS) aproximadamente 7 milhões de crianças no mundo estão infectadas por algum tipo de parasitose (Santos, 2019).

O diagnóstico laboratorial da ascaridíase se dá através do Exame Parasitológico de Fezes (EPF), onde é pesquisado a presença de ovos do Ascaris lumbricoides através de métodos tais como: exame direto a fresco, método de Hoffman, método de Kato-Katz, entre outros (Andrade Junior et al, 2020). O Método Hoffman se fundamenta na sedimentação espontânea e permite a concentração de ovos, cistos, oocistos e larvas de inúmeras espécies por meio de uma sedimentação gravitacional de uma amostra fecal, é caracterizado como uma técnica qualitativa, tida como principal método de investigação usual e no cotidiano laboratorial pelo seu baixo custo (Lima et al., 2020). Já o Exame Direto a Fresco, permite a identificação de protozoários (trofozoítos e cistos) e de helmintos (ovos, larvas e pequenos adultos (Lucena, 2017).

O presente estudo visa apresentar conceitos, interpretações e reflexões do termo sensibilidade encontrados na literatura, em documentos científicos. Assim, se justifica pelo fato de que para procedimento padrão das técnicas de diagnóstico parasitológico geralmente é utilizado a sensibilidade para comparação e verificação de desempenho de testes, cujo o critério fundamental para sua utilidade é se o mesmo agrega informação a um nível além daquele já existente, ou seja, 
normalmente partem do conhecimento prévio se há ou não a doença, se o diagnóstico já existe ou de que o mesmo já foi descartado, portanto seus conceitos tornam-se limitados.

Portanto, o objetivo geral é comparar a sensibilidade dos métodos laboratoriais direto a fresco e de Hoffman na área de Parasitologia. E os objetivos específicos são: explanar sobre os métodos realizados para diagnostico em análise parasitológicas; explanar sobre os métodos realizados para diagnostico em análise parasitológicas e descrever o conceito de sensibilidade aplicado nesses métodos.

\section{Metodologia}

Trata-se de uma revisão bibliográfica sobre a sensibilidade dos métodos Direto à Fresco e Hoffman como objetivo de compreender o tema em estudo. A abordagem será quantitativa, os métodos quantitativos têm o objetivo de mostrar dados, indicadores e tendências observáveis, ou produzir modelos teóricos abstratos com elevada aplicabilidade prática. Suas investigações evidenciam a regularidade dos fenômenos.

Neste tipo de pesquisa essa técnica traz consigo uma forma de analisar as pesquisas realizadas através dos resultados obtidos, e podem estar em materiais como artigos de revistas. Para este trabalho, serão seguidas as seis fases da Revisão Integrativa, que consiste em: elaboração da pergunta norteadora; busca ou amostragem na literatura, discussão dos resultados, análise crítica dos estudos incluídos, coleta de dados e apresentação da revisão integrativa (Appolinário, 2016).

A busca de artigos científicos foi realizada nas bases de dados eletrônicas: Scientific Electronic Library Online (SCIELO), Literatura Latino-Americana em Ciências da Saúde (LILACS), National Library of Medicine and the National Institute of Health (Pubmed).

Foram incluídas publicações de 2015 a 2021, em idiomas português, espanhol e inglês disponíveis na íntegra para análise, e foram excluídos os não disponíveis na íntegra, estudos com datas anteriores a 2015, e que estavam fora do contexto, exceto livros e diretrizes.

Após a revisão de literatura prosseguiu-se com a seleção dos artigos teses, dissertações e documentos. Estes materiais foram selecionados e separados por assunto conforme a relevância do tema que se propõe a investigar. Feito isso, procedeu à leitura exaustiva dos materiais a serem analisados.

O total de artigos científicos consultados para a realização dos objetivos propostos na presente pesquisa, à revisão foram realizados com 100 artigos, descartados 70 artigos, e utilizados 30 artigos publicados a partir do ano 2015 ao ano de 2021, tratavam do tema desenvolvido no artigo. 
Figura 1 - Fluxograma da pesquisa.

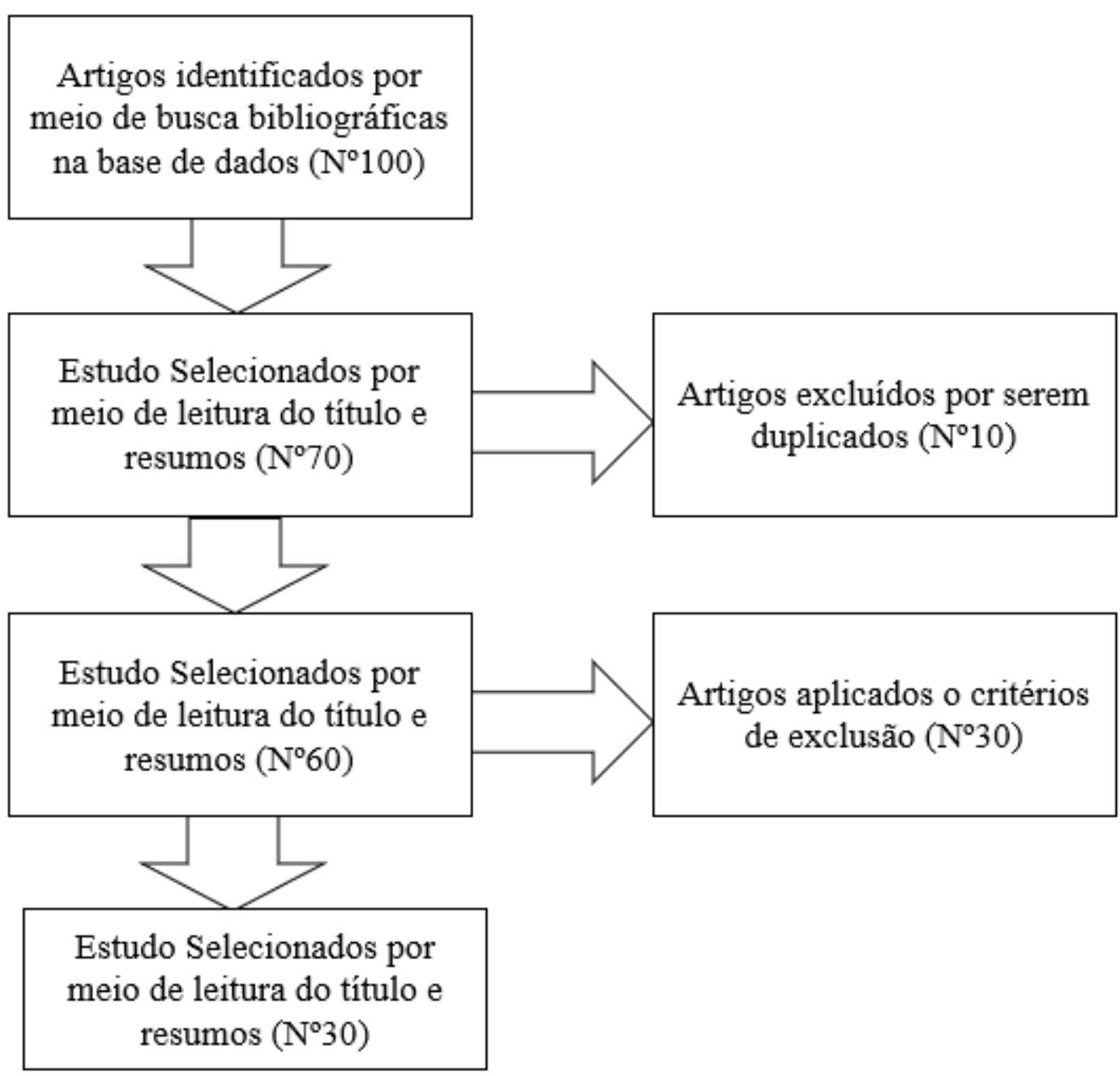

Fonte: Autores (2021).

\section{Resultados e Discussão}

\subsection{Conceituando Sensibilidade}

Sendo o diagnóstico clínico das parasitoses intestinais impreciso por se basear em uma história clínica que pode ser, muitas vezes, assintomática ou totalmente variada, o diagnóstico laboratorial é de extrema importância para diagnosticar as infestações, sendo crucial na definição da conduta terapêutica adequada (Azevedo et al, 2017).

A qualidade dos testes diagnósticos é fundamental e inúmeras pesquisas são feitas com a finalidade de melhorar as técnicas, pois o resultado pode ser "positivo" ou "negativo", no entanto, é preciso considerar a possibilidade de o teste ter dado resultado errado. Portanto os parâmetros avaliados para estimar a probabilidade de ocorrerem falsos-positivos ou falsosnegativos em testes diagnósticos são, principalmente, sensibilidade e especificidade (Vieira, 2018).

A princípio é notório a dificuldade de encontrar os conceitos de sensibilidade e especificidade em estudos. Segundo Ferreira e Patino (2017) essas são medidas importantes porque nos fornece a idéia de quão bom é o desempenho de um novo teste diagnóstico em comparação a um teste padrão ouro existente. As autoras definem sensibilidade como uma proporção de indivíduos que têm a doença e apresentam teste positivo, conceito que vai de acordo com Silva, Amorim e Fiaccone (2016), que conceituaram a sensibilidade como a porcentagem das pessoas com diagnóstico que apresentam positividade para o teste, isto é, se de 100 pessoas com um diagnóstico, 80 tem positividade para um teste, a sensibilidade deste é de $80 \%$. 
A respeito do termo especificidade Souza (2016), conceitua como uma proporção de indivíduos que não tem a doença e apresentam teste negativo, e Pinheiro et al. (2021) em termos técnicos define especificidade como a porcentagem das pessoas sem o diagnóstico que apresentam negatividade para o teste, ou seja, se de 100 pessoas sem um diagnóstico, 80 tem negatividade para um certo teste, a especificidade deste é de $80 \%$.

Dessa forma, estudos mostram que sensibilidade e especificidade não andam juntos, quando há um aumento da sensibilidade consequentemente leva a queda de especificidade, da mesma maneira acontece com o aumento da especificidade, ou seja, dificilmente um teste diagnóstico é considerado $100 \%$ de sensibilidade e especificidade, sobretudo existem limitações na utilização dos resultados de um teste, onde aprender a interpretar as propriedades destes testes são importantes medidas para avaliação de seu desempenho (Patino \& Ferreira, 2017).

\subsection{Epidemiologia}

No Brasil existe uma elevada prevalência de parasitoses intestinais que constituem um grande problema de saúde pública e estudos acerca da patologia são fundamentais nos dias atuais (Lins, 2016). As precárias condições de habitação, a ausência de saneamento básico, os hábitos higiênicos inadequados, o baixo nível educacional e cultural das populações que vivem em zonas rurais e periféricas das grandes cidades contribui para a perpetuação do problema (Prado et al., 2021). A esse respeito Abreu, Teston, Paula e Ribas (2015), ressaltam que determinados parasitas, representam um agravante problema de saúde pública, caracterizando síndromes de má nutrição, deficiência de aprendizado e incapacidade funcional, principalmente em crianças.

A estimativa é de que haja mais de 3,5 bilhões de pessoas infectadas no mundo por alguma espécie de parasita intestinal com a maior prevalência em países em desenvolvimento. O Brasil apresenta altas taxas de indivíduos nessa condição em diferentes regiões, com maior prevalência nas regiões Norte e Nordeste (Marques et al. 2021). A alta prevalência se dá em regiões com condições de vida e de saneamento básico insatisfatórias, uma vez que a contaminação ocorre predominantemente por via fecal-oral, pela ingestão de cistos e ovos, em água e alimentos contaminados, persistindo assim, grande impacto na morbidade dos pacientes (Melo et al., 2021).

A maioria dos casos notificados decorre da aglomeração populacional em ambientes impróprios, com escassez de higiene falta de conhecimento sobre medidas preventivas e profiláticas. A literatura aponta como parasitas mais comuns na infestação dessa população os Ascaris lumbricoides, Entamoeba histolytica, Enterobius vermicularis, Trichuris trichiura e Giardia lamblia (Olímpio et al., 2019).

Dentre as alterações biológicas de grande importância que as parasitoses podem causar estão a anemia e distúrbios intestinais, fatores estes que podem culminar em uma desnutrição consequente da má absorção disponibilizada pela presença de enteroparasitas que atuam como fatores co-adjuvantes para manutenção ou agravamento deste tipo de quadro clínico (MENEZES JRet al., 2020). Isso demonstra o nível de atenção que se deve voltar a esses acometimentos que muitas das vezes ainda é negligenciada (Abreu et al., 2021).

\subsection{Método Direto a Fresco e Hoffman}

As doenças parasitárias estão relacionadas às populações vulneráveis pela precariedade sanitária e à pobreza. Somente por meio de investigações laboratoriais é possível definir se o paciente está ou não infectado, e o EPF constitui-se como um importante procedimento laboratorial de análises clínicas, pois indicará o nível de parasitismo bem como o tratamento específico para cada infecção (Silva et al., 2020). É relativamente rápido e de baixo custo, e os procedimentos são muito simples (Ferreira, 2019), mas para Chielle et al. (2020), novas metodologias para o diagnóstico parasitológico são necessárias e que estas ofereçam bons parâmetros de sensibilidade, especificidade, baixo custo, rapidez e reprodutibilidade. 
Apesar da existência de inúmeras técnicas, quantitativas e qualitativas, proposta para o EPF no laboratório de rotina preconiza-se uma combinação de técnicas com o objetivo de aumentar a eficácia diagnóstica e diminuir os resultados falsos negativos, a comparação de técnicas tem sido estudada por diversos autores ao longo dos anos, objetivando avaliar sua eficiência no diagnóstico parasitológico. A escolha da técnica dependerá do grau de confiabilidade e de sensibilidade do método, além de necessitar de recursos menos onerosos (Vieira, 2018).

O exame direto a fresco e a técnica de Hoffman, Pons e Janer ou Lutz (HPJL) são os métodos laboratoriais mais utilizados, pois estas técnicas têm como principal vantagem a necessidade mínima de materiais e recursos financeiros, porém a desvantagem é de apresentar uma grande quantidade de detritos fecais no sedimento, dificultando a preparação e o exame microscópico (Lima, 2016).

O exame parasitológico direto a fresco é considerado um procedimento simples e eficiente para estudo das fezes que permite o diagnóstico dos protozoários (trofozóitos e cistos) e dos helmintos (ovos, larvas e pequenos adultos). A técnica realizada por meio de uma gota de soro fisiológico (salina) e uma pequena porção de fezes depositados sobre uma lâmina e diluída com um palito de modo que fique homogêneo e transparente (figura 2), representando assim uma prática simples, porém eficaz (Silva, 2018).

Figura 1 - Procedimento do teste direto a fresco.

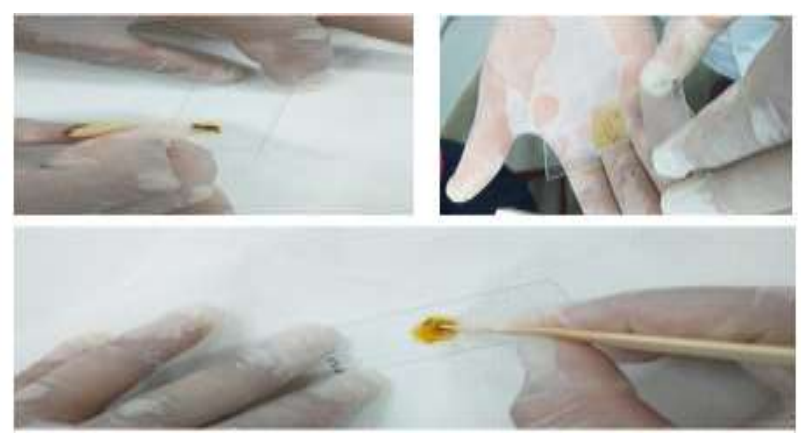

Fonte: Autores (2021).

De acordo com Lima (2018), o método de sedimentação espontânea é o método qualitativo mais utilizado nos laboratórios de análises clínicas, por ser de fácil execução e baixo custo, apresentar boa sensibilidade, não exigir aparelhagem especial e permitir o diagnóstico simultâneo de parasitoses. Também conhecido como método de sedimentação, considerado padrão ouro para diagnóstico, consiste na combinação da gravidade e da sedimentação, indicada para a pesquisa de ovos e larvas de helmintos e cistos de protozoários.

Ao contrário de outras técnicas de concentração, requer grande quantidade de material fecal (Figura 2), garantindo assim maior segurança e eficácia do diagnóstico, porém há desvantagem relacionada à grande quantidade de detritos fecais presente no sedimento, o que dificulta a preparação do esfregaço e a visualização da lâmina (Pedrosa et al, 2020). 
Figura 2 - Procedimento do método de Hoffman.

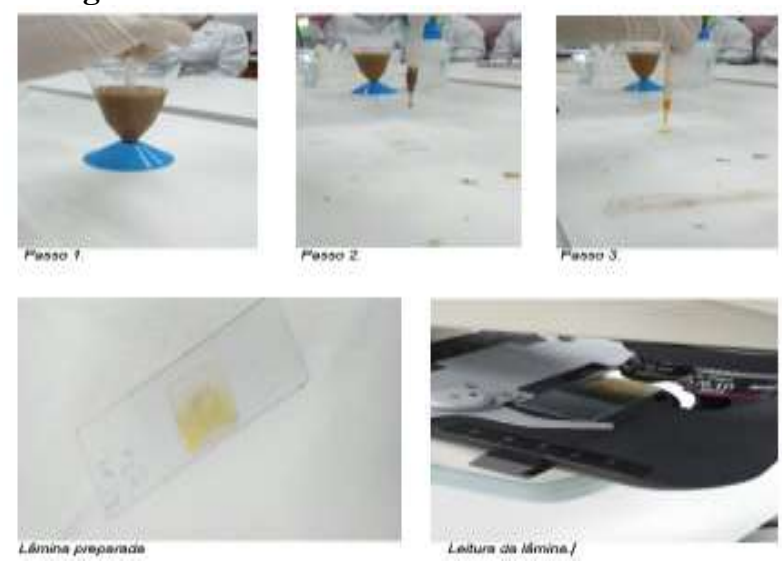

Fonte: Autores (2021).

Enfim, as combinações dos métodos laboratoriais específicos tornam-se necessários nos exames parasitológicos para detectar por meio direto associado a microscopia e informação dos sintomas do paciente o tipo de infecção intestinal. Dos 30 artigos incluídos neste estudo, foram apenas 4 que tiveram realmente o processo de tratamento de ambos para comparação.

O Quadro 1. Demonstra os principais resultados quanto à sensibilidade do Exame Direto a Fresco e do Método de Hoffman.

Quadro 1 - Resultado da sensibilidade em comparação do método a fresco e Hoffman para Ascaris lombricóides.

\begin{tabular}{|c|c|c|}
\hline AUTOR/ANO & TÍTULO & RESULTADO \\
\hline Lima, 2018. & $\begin{array}{l}\text { Prevalência de enteroparasitoses em } \\
\text { usuários do Laboratório de Análises } \\
\text { Clínicas do Hospital Universitário } \\
\text { Ana Bezerra, Santa Cruz - RN. }\end{array}$ & $\begin{array}{l}\text { O método Hoffman tem uma boa sensibilidade para ovos de helmintos, porém quando comparado } \\
\text { com o método da centrífugo-flutuação (Faust et al.) é evidenciada a maior sensibilidade do } \\
\text { método de Faust et al. para cistos de protozoários. O método de Faust et al. complementar ao HPJ, } \\
\text { melhora significativamente os resultados do diagnóstico. }\end{array}$ \\
\hline Silva et al., 2020. & $\begin{array}{l}\text { Perfil de desempenho de técnicas } \\
\text { coproscópicas Coproplus } ® \text { e } \\
\text { Hoffman, Pons e Janner no } \\
\text { diagnóstico de giardíase. }\end{array}$ & $\begin{array}{l}\text { A técnica Coproplus }{ }^{\circledR} \text { indicou sensibilidade de } 94 \% \text { (intervalo de confiança: } 89-96 \% \text { ). O método } \\
\text { de Hoffman apresentou sensibilidade de } 98 \% \text { (intervalo de confiança: } 95-99 \% \text { ). A comparação dos } \\
\text { métodos, constou que a diferença de sensibilidade é de } 4 \% \text {; o que dá a ideia da funcionabilidade } \\
\text { de ambas metodologias, de modo que os métodos são aprazíveis dentro das aplicações } \\
\text { laboratoriais. }\end{array}$ \\
\hline $\begin{array}{l}\text { Munareto et al, } \\
2021\end{array}$ & $\begin{array}{l}\text { Parasitoses em crianças na fase pré- } \\
\text { escolar no Brasil: revisão } \\
\text { bibliográfica. }\end{array}$ & $\begin{array}{l}\text { O exame direto a fresco é muito utilizado para pesquisa dos principais helmintos e protozoários } \\
\text { intestinais em humanos, porém apresenta baixa sensibilidade, pois não utiliza um processo para a } \\
\text { concentração das formas parasitárias, o que pode mascará-las. }\end{array}$ \\
\hline Gomes, 2019. & $\begin{array}{l}\text { Análise parasitológica de pré- } \\
\text { escolares de escolas municipais e da } \\
\text { comunidade ribeirinha pertencente ao } \\
\text { município de São Paulo De } \\
\text { Olivença-AM. }\end{array}$ & $\begin{array}{l}\text { O exame direto a fresco demonstrou uma alta sensibilidade na pesquisa principalmente da } A \text {. } \\
\text { lumbricoides, porém sempre é recomendado que se use outro método complementar a fim de se } \\
\text { aumentar a confiabilidade do diagnóstico. }\end{array}$ \\
\hline
\end{tabular}

Fonte: Autores (2021).

\section{Considerações Finais}

O exame direto a fresco e a técnica de Hoffman são os métodos mais utilizados nos laboratórios de análises clínicas, dada a sua simplicidade, baixo custo e necessidade de poucos instrumentos. A literatura aconselha fazer uso de 3 ou mais técnicas laboratoriais, principalmente na pesquisa de parasitoses, pois nenhuma técnica apresenta $100 \%$ de sensibilidade a esses estudos. Direto a fresco e Hoffman, quando utilizados em conjunto aumentam as probabilidades de diagnósticos reais e confiáveis, principalmente no diagnóstico de ovos e cistos de helmintos, porém separados cada um possui maior sensibilidade em casos específicos. Pela escassez de estudos comparativos destes dois métodos, fica evidente e vale a sugestão de mais estudos nesse sentido, uma vez que estes métodos se apresentam tão importantes no diagnóstico das parasitoses, que têm se tornado uma questão de saúde pública, porém pelo difícil diagnóstico acaba sendo negligenciada no nosso dia a dia.

Para pesquisas futuras pode-se realizar um estudo transversal com outros laboratórios da região central de Manaus 
sobre aplicabilidade dos métodos laboratórios de análises clínicas desenvolvendo assim pesquisa que amplia o conhecimento sobre probabilidades de diagnósticos reais e confiáveis.

\section{Referências}

Abreu, A. P. D., Teston, A. P. M., Paula, C. A. D., \& Ribas, A. D. (2015). Epidemiologia das parasitoses intestinais em pré-escolares e escolares de duas creches em Marialva-Pr In: IX EPCC - Encontro Internacional de Produção Científica UniCesumar. 9, 4-8.

Andrade Júnior, F. P., Alves, T. W. B., \& de Arruda Barbosa, V. S. (2020). Ascaridíase, himenolepíase, amebíase e giardíase: uma atualização. Educação, Ciência e Saúde, 7(1).

Antunes, A. S., \& Libardoni, K. S. D. B. (2017). Prevalência de enteroparasitoses em crianças de creches do município de Santo Ângelo, RS. Revista Contexto \& Saúde, 17(32), 144-156.

Appolinário, P. Metodologia de ciência: filosofia e prática da pesquisa. (2a ed.) Congange Leraning, 2016.

Azevedo, E. P., Almeida, E. M., Matos, J. S., Ramos, A. R., Siqueira, M. P., Fonseca, A. B. M., ... \& Uchôa, C. M. A. (2017). Diagnóstico parasitológico em amostras fecais no laboratório de análises clínicas: comparação de técnicas e custo de implantação. Revista Brasileira de análises clínicas, 49 (4), 401-407.

Chielle, E. O. (2020). Desenvolvimento de sistema estruturado com inteligência artificial para apoio no diagnóstico de parasitoses intestinais. Clinical \& Biomedical Research, 40(3).

Euzébio, R. (2020). A infecção pelo Trichomonas vaginalis e suas possíveis relações com a aquisição e transmissão do vírus HIV. Revista Científica Faculdade Unimed, 2(1), 96-111.

Ferreira, A. L. D. A. (2019). Exame parasitológico de fezes de rotina: métodos utilizados em laboratórios de análises clínicas da rede pública em Natal, Rio Grande do Norte. Trabalho de Conclusão de Curso (Bacharel em Farmácia) - Universidade Federal do Rio Grande do Norte. Natal.

Ferreira, J. C., \& Patino, C. M. (2017). Entendendo os testes diagnósticos. Parte 1. Jornal Brasileiro de Pneumologia, 43, 330-330.

Garcia, F. K. C., Ribeiro, J. F., Barbosa, J. H. R., Brito, L. L. R., de Souza, L. O., Olímpio, L. T., \& da Costa, M. E. V (2019). Incidência de protozoários e helmintos no exame parasitológico realizado no laboratório central de biomedicina no primeiro semestre de 2018. Medicina e Biomedicina; 2 , $107-112$.

Gomes, S. R. (2019). Análise parasitológica de pré-escolares de escolas municipais e da comunidade ribeirinha pertencente ao município de São Paulo de Olivença-AM. Trabalho de Conclusão de Curso (Licenciatura em Ciências Biológicas) - Centro de Estudos Superiores de Tabatinga-Am. Universidade do Estado do Amazonas. Tabatinga: 2016.

Lima, E. C. D. S. (2018). Prevalência de enteroparasitoses em usuários do laboratório de análises clínicas do Hospital Universitário Ana Bezerra, Santa CruzRN. Trabalho de Conclusão de Curso (Bacharel em Farmácia) - Universidade Federal de Campina Grande.Cuité: 2018

Lima, F. L. O., Santos, C. S. C. D., Almeida, F. C. D., Rocha, L. S., \& Lima, A. G. D. (2020). Um século do exame parasitológico de Lutz e sua relevância atual. Revista Brasileira de Análises Clínicas, 32-34.

Lima, J. A. S. (2016). Comparação da prevalência de parasitos entéricos em gatos errantes e domiciliados em Goiânia-Goiás, análise da acurácia de técnicas parasitológicas e avaliação da copro-PCR para o diagnóstico de Toxoplasma gondii. Dissertação (Mestrado em Medicina Tropical e Saúde Pública) Universidade Federal de Goiás, Goiânia.

Lins, I. V. F., Soares, C. V. D., Costa, R., Soares, G. V. D., Duarte, A. B. S., \& dos Santos Medeiros, J. (2016). Comparação entre o método da fita adesiva e o método de sedimentação espontânea para o diagnóstico de Enterobius vermicularis. Journal of Biology \& Pharmacy and Agricultural Management, 12(4).

Lucena, G. T. D. S. (2017). Levantamento epidemiológico de zoonoses parasitárias em assentamento rural na microrregião de Patos-PB, Brasil. Dissertação (Mestrado em Zootecnia) - Centro de Saúde e Tecnologia Rural. Centro de Saúde e Tecnologia Rural.

Marques, J. R. A., Gutjahr, A. L. N., \& de Souza Braga, C. E. (2021). Prevalência de parasitoses intestinais em crianças e pré-adolescentes no município de Breves, Pará, Brasil. Saúde e Pesquisa, 14(3), 1-18.

Melo, T. D. S. S., dos Santos, W. B., \& de Faria Castro, C. L. (2021). Caracterização do perfil epidemiológico das parasitoses no município de São João DelRei, Minas Gerais, BrasiL. In Anais do Congresso de Pesquisa e Extensão do UNIPTAN, 5, 94-104.

Menezes Júnior, R. C., de Lima Junior, C. A. A., Marinho, I. G., de Melo Braga, K. H., do Nascimento, R. O., dos Santos Calandrini, T. D. S., ... \& de Oliveira Menezes, R. A. (2020). Enteroparasitoses, anemia e estado nutricional de uma população ribeirinha no estado do Amapá. Revista Eletrônica Acervo Saúde, 12(5), e2841-e2841.

Munareto, D., de Lima, A. P. S., Zardeto-Sabec, G., \& Vieira, S. L. V. (2021). Parasitoses em crianças na fase pré-escolar no Brasil: revisão bibliográfica. Research, Society and Development, 10(1), e1910111195-e1910111195.

Patino, C. M. \& Ferreira, J. C (2017). Entendendo os testes diagnósticos. Parte 1. Jornal Brasileiro de Pneumologia, 43, 330-330.

Pinheiro, P. C., Barros, M. B. D. A., Szwarcwald, C. L., Machado, Í. E., \& Malta, D. C. (2021). Differences between self-reported and laboratory measures of diabetes, chronic kidney disease, and hypercholesterolemia. Ciência \& Saúde Coletiva, 26, 1207-1219.

Pinto, L. C., Gonçalves, M. N. L., Viana, M. W. C., do Nascimento, M. P., Candido, A. S., \& Ferreira, R. J. (2018). Estruturas parasitárias em alface (Lactuca sativa 1.), comercializadas na feira livre do município de Jardim, Ceará. Cadernos de Cultura e Ciência, 17(1), 1-14. 
Research, Society and Development, v. 10, n. 15, e496101523460, 2021

(CC BY 4.0) | ISSN 2525-3409 | DOI: http://dx.doi.org/10.33448/rsd-v10i15.23460

Prado, T., Azevedo, P., Almeida, V., Silva, M. M., \& Oliveira, H. (2021). Educação em saúde: visita domiciliar e avaliação epidemiológica em parceria com o programa de saúde da família. Enciclopédia biosfera, 18(37).

Rodrigues, A. G., do Rosário Palma, A. L., Miao, A. V., Kawasaka, A. K., da Silva, B. P. M., Pedrosa, E. L. C., \& de Lapena, S. A. B. Avaliação de técnicas quantitativas e qualitativas no diagnóstico de parasitologia. Ciências biológicas [recurso eletrônico]: campo promissor em pesquisa. - Ponta Grossa, PR: Atena Editora, 2020.

Santos, C. E. P. D. (2019). Prevalência de Ascaris lumbricoides avaliados no município de São FelipeBA. Monografia (Bacharel em farmácia) - Faculdade Maria Milza.

Silva, C. P., da Silva Aranda, K. R., Barnabe, A. S., de Mello, T. R. D. C., \& Ferraz, R. R. N. (2020). Perfil de desempenho de técnicas coproscópicas Coproplus® e Hoffman, Pons e Janner no diagnóstico de giardíase. Revista de Epidemiologia e Controle de Infecção, 10(2), 181-184.

Silva, N. B., Amorim, L. D. A. F., \& Fiaccone, R. L. (2016). Sensibilidade E Especificidade De Testes Diagnósticos Em Diferentes Cenários De Dependência. Revista Brasileira De Biometria, 34(3), 489-506.

Teixeira, A. G. S., Santos, F. B., Santos, G. R., de Sousa Santos, M. D. R., \& Rodrigues, G. M. (2019). Os efeitos do saneameto básico precário para o aumento da ascaris lumbricoides. Revista Brasileira de Pesquisa em Ciências da Saúde, 5(10), 34-40.

Souza, T. D. F. D. (2016). Especificidade e sensibilidade do Questionário de Transtorno do Desenvolvimento da Coordenação-Brasil para crianças de 8 a 10 anos. Dissertação (Mestrado Terapia Ocupacional) - Programa de Pós-graduação em Terapia Ocupacional da Universidade Federal de São Carlos. São Carlos: 2017.

Vieira, S. (2010). Bioestatística: tópicos avançados. In Bioestatística: tópicos avançados. (4a ed.) Elsevier. 